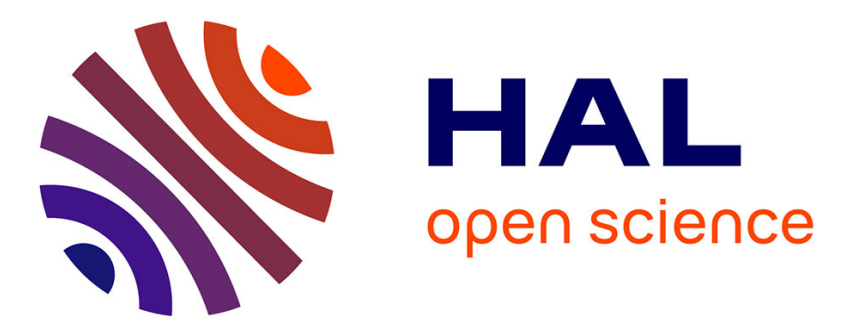

\title{
Fouilles à la citadelle méridionale de Forum Julii (Fréjus, Var) en 1955 \\ Paul-Albert Février
}

\section{To cite this version:}

Paul-Albert Février. Fouilles à la citadelle méridionale de Forum Julii (Fréjus, Var) en 1955. Gallia - Fouilles et monuments archéologiques en France métropolitaine, 1956, 14 (1), pp.35-53. 10.3406/galia.1956.1454 . hal-01923580

\section{HAL Id: hal-01923580 \\ https://hal.science/hal-01923580}

Submitted on 4 Feb 2020

HAL is a multi-disciplinary open access archive for the deposit and dissemination of scientific research documents, whether they are published or not. The documents may come from teaching and research institutions in France or abroad, or from public or private research centers.
L'archive ouverte pluridisciplinaire HAL, est destinée au dépôt et à la diffusion de documents scientifiques de niveau recherche, publiés ou non, émanant des établissements d'enseignement et de recherche français ou étrangers, des laboratoires publics ou privés.

\section{(ㅇ)(1) $\$$}

Distributed under a Creative Commons Attribution - NonCommercial - NoDerivatives| 4.0 


\title{
FOUILLES A LA CITADELLE MÉRIDIONALE DE FORUM JULII (FRÉJUS, VAR) EN 1955
}

\author{
par M. Paul-Albert FÉvrier
}

Au sud de la ville de Fréjus (fig. 1), la plaine de l'Argens est facilement surveillée d'une butte appelée Butle Saint-Antoine, dont l'altitude moyenne est aujourd'hui de 10 mitres (fig. 2). La base de cette butte est constituée de grès permiens, sur lesquels un sondage a montré la présence d'argile blanche plaisancienne. A l'est, au sud et à l'ouest, une plaine d'alluvions "récentes". Vers le nord, la butte était rattachée aux banc's de grès qui portent la ville gallo-romaine ; l'installation de la voie de chemin de fer a sensiblement modifié ce quartier, en créant une large percée. De tous les côtés, cette butte est entourée de murailles antiques, que les historiens de Fréjus ont considérées comme celles d'une des citadelles qui protégeaient le port. L'autre citadelle est la Plate-Forme qui a commencé d'être fouillée par le Dr A. Donnadieu ${ }^{1}$; la butte Saint-Antoine, depuis les travaux de Ch. Texier, n'avait fait l'objet d'aucune recherche systématique et seules des découvertes fortuites ont été signalées ${ }^{2}$.

\section{I. - L'ENCEINTE}

Les murs de la citadelle ont la forme d'un hexagone irrégulier (fig. 3). Deux des côtés, les faces est et ouest, sont plus longs que les autres; le premier mesure 108 mètres et le second 145 mètres environ. Le mur nord n'a que 81 mètres et les trois autres, vers le sud, 30, 25) et 50 mètres environ. A trois des angles vers le sud-est, des tours rondes.

C'est d'abord au revers de ces murs, à l'intérieur de la citadelle, qu'ont été menés les travaux ${ }^{3}$. Le mur est (A du plan fig. 3, et fig. 2) dominait le port ; sa base

(1) Forma orbis Romani, Carte archéologique de la Gaule romaine, II, 1932, no 4, p. 2 et 3; Les fouilles récentes du "Forum .Julii "à Fréjus, dans Congrès arch. de France, XCV le session tenue à Ai.t-en-Pronence et à Nice, 1932, p. 264-76; Les fouilles du Praetorium de l'arsenal natal du F. J., dans Comples rendus de l'Ac. des Inscr. et Belles-Leltres, 1932, p. 115-118; Fréjus, le port militaire du F. J., Paris, 1935, plan ; Le tribunal et la chapelle des aigles du Prétoire de Fr., dans Bull. arch. du com., 1938-40, p. $477-80$.

(2) Corp. inscr. lat., XII, 5733 : P.-A. Fíritr, Récentes découverles de Fréjus, dans Provence hist., III-1952, p. 71-74.

(3) Ces fouilles ont été entreprises à la suite d'une demande de lotissement. Aussi a-t-il paru 
PAUL-ALBERT FÉVRIER

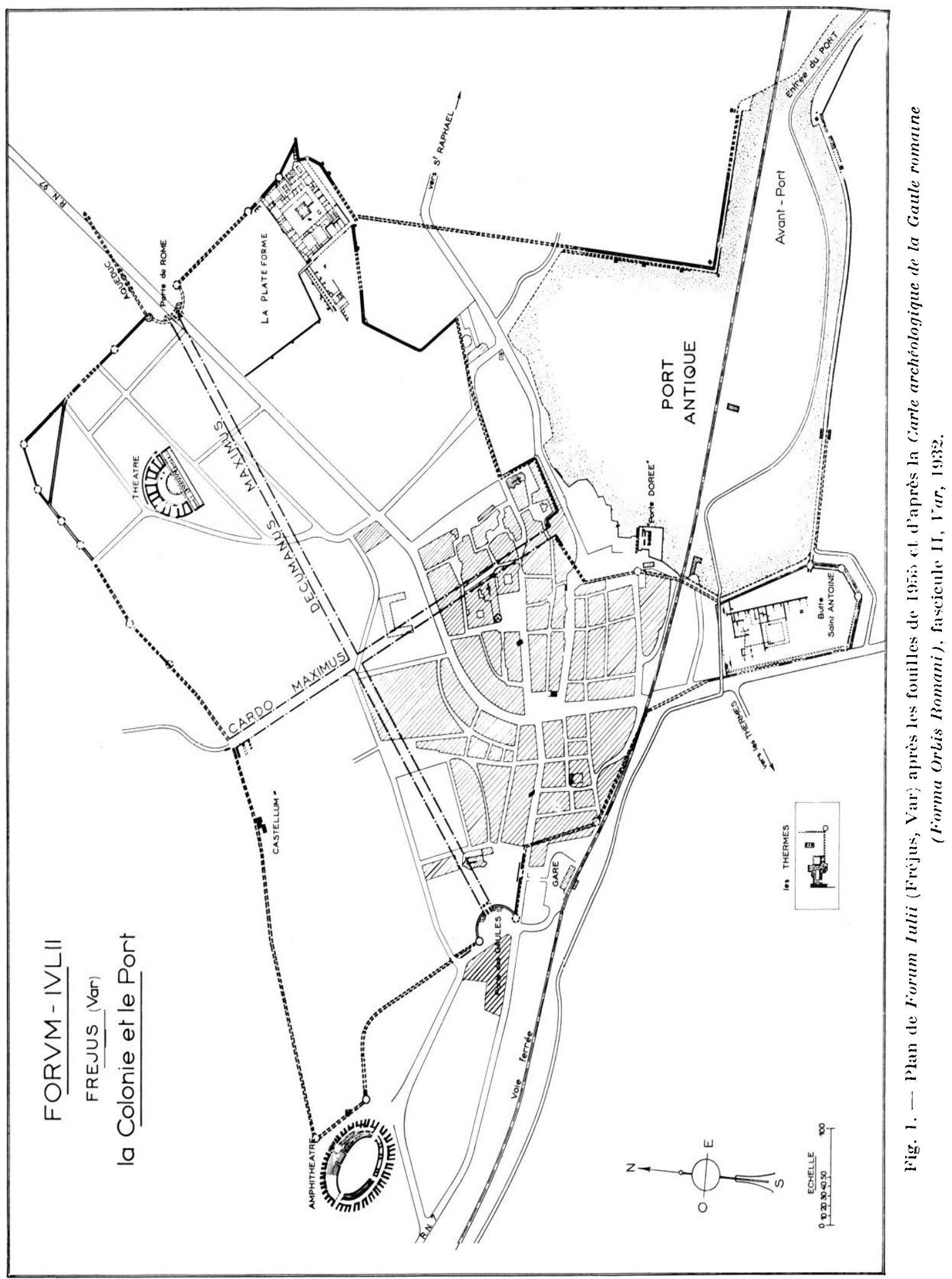


est aujourd'hui cachée par les terres qui ont comblé ce port. La muraille est constituée d'un blocage très compart, épais au sommet de $2 \mathrm{~m}, 90$ et s'élargissant vers la base. Ce mur n'était parementé que vers l'extérieur; les moellons sont hauts de ()m,15 a 0 m,20 et les joints sont épais de 1 a 2 rentimètres; détail caractéristique : rertains moellons ne sont pas posés à plat. mais de champ. Ce mur portait un rhemin de ronde large de $2 \mathrm{~m}, 30$ et bordé du côté du port d'un parapet de $0 \mathrm{~m} .60$ d'épaisseur. Ce parapet s'est conservé aux points fouillés

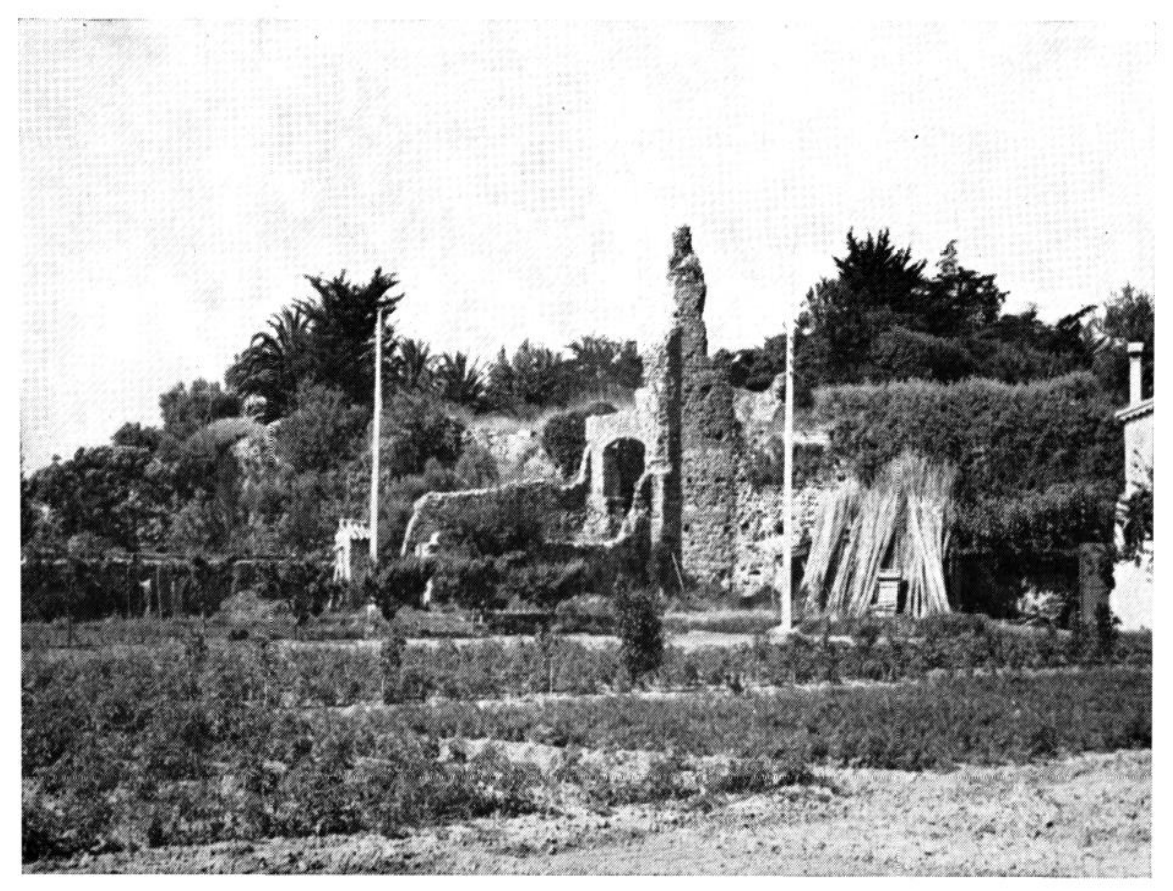

l.ig. 2. - - I.a Butte Saint-Antoine, vue de l'ancien port. A gauche, le naur oriental (A du plan dig. 3 ; ; au rentre, la tour dite "phare".

sur une hauteur de $0 \mathrm{~m}, 90$, mais aucune trace de créneaux n'a été relevée ; peutêtre avaient-ils leur base à un niveau supérieur. Le parement du parapet est identique à celui du mur sur lequel il repose; il peut donc dater de la mème époque. En quelques points, ce mur d'enceinte s'appuie contre les banc's de grès de la butte, par exemple près de la tour appelée "phare ". En d'autres points, les sondages n'ont mis au jour que des terres rapportées à soll revers.

Le seul objet recueilli a élé un fragment de vase décoré d'Arezzo, qui a été trouvé

nécessaire d'étendre le plus possible la surface de fouille. Je tiens i remercier de leur aide II. l'alı!r' R. Boyer, II. J. Gourvest, II. (i. Giger, architecte I). I. L. G., ainsi quue l'entrepreneur II. A. Ghis et son éfuipe d'ourriers nord-africains. Les travaux ont éti menes sous la surveillance de II. F. Benoit qui m'a fait bénéficier de ses conseils. 
i $0^{\mathrm{m}}, 20$ au-dessous du niveau du chemin de ronde. Ce vase de la forme 11 de Iragendorff est orné du motif répété de la danseuse au kalathos, tournée vers la gauche et dont le bras gauche est tendu vers l'arrière. Il semble bien sortir de l'atelier de Perennius ${ }^{4}$. Mais ayant été découvert en dehors de toute stratigraphie, il ne peut servir d'élément de datation (fig. 4).

A l'extrémité méridionale du mur $A$, se dresse une tour de $7 \mathrm{~m}, 20$ de diamètre intérieur, appelée traditionnellement le "phare ". A l'étage inférieur, elle était percée de quatre portes : celle du nord communiquait sans doute avec le chemin de ronde décrit plus haut. grâce à un escalier ; celle de l'ouest ouvrait vers l'intérieur de la citadelle; celle de l'est permettait de gagner le quai, soit par un escalier. soit par un plan incliné ; la quatrième, enfin, donnait accès à la partie méridionale de l'enceinte. Cette lour avait un étage dont il ne reste qu'un faible pan de mur, percé de part en part de trous de boulins. Le plan de Texier montre à l'extérieur un escalier qui servait peut-être d'accès aux étages ${ }^{5}$.

Les trois murs sud (B, C et D du plan fig. 2) n'ont pu être étudiés au cours de cette campagne. L'appareil est identique à celui du côté oriental mais la technique de construction est différente. Deux d'entre eux, B et $C$, sont formés a la base d'un massif de blocage. Au dessus, courait un couloir voûté qui rommuniquait aver l'étage inférieur des tours, et qui supportait le chemin de ronde. Le mur D a, dans sa partie orientale, a la place du couloir, une série de niches carrées qui ont peut-être joué le même rôle que les niches semicirculaires du mur ouest; dans la partie occidentale, il y eut un aqueduc, dont subsiste le départ et le fond en bélon de tuileau. Il est impossible de savoir si toutes ces dispositions remontent à l'origine, d'autant que diverses modifications ont été apportées à celle construction : c'est ainsi que le mur $C_{i}$ a été percé d'une large ouverture qui donne accès à une salle voûtée, au fond de laquelle est ménagé un puits. ${ }^{6}$. Or ce dispositif n'est pas primitif, car l'appareil est très différent de celui du mur C ; les moellons n'ont que 7 à 9 centimètres de hauteur et sont longs de 20 a 30 . Devant cette salle, Texier avait fouillé une maison ?.

Le mur occidental (E du plan fig. 3) n'a pas été fouillé mais ses dispositions sont

(4: A. OxÉ, Arretinische Reliefgefüsse vom Rhein, Francfort, 1933, pl. XX, 95 ; XLI, 146 ; LXII ; et p. 28-33 (ț̣pes légèrement différents). I. Dragiendorff, Arretinische Relieflieramili... ergänzt... von $K$. Walzinger, Reutlingen, 1948, p. 55-61 et pl. I, 1-4.

(5) Ch. Texule, .Mém. sur la ville el le port de Fr., dans Mém. prés. par div. sav. à l'Ac. des Inscr. ef Belles-Lettres, $2^{\mathrm{e}}$ série, II-1849, pl. V et p. 198. p. 477 .

(6) Dessin dans V. Petr', Fréius, Cannes, p. 90. J.-A. Auberas, IIisl. de Fr., Fréjus, 1881,

(7) Art. cit., p. 207-8. 


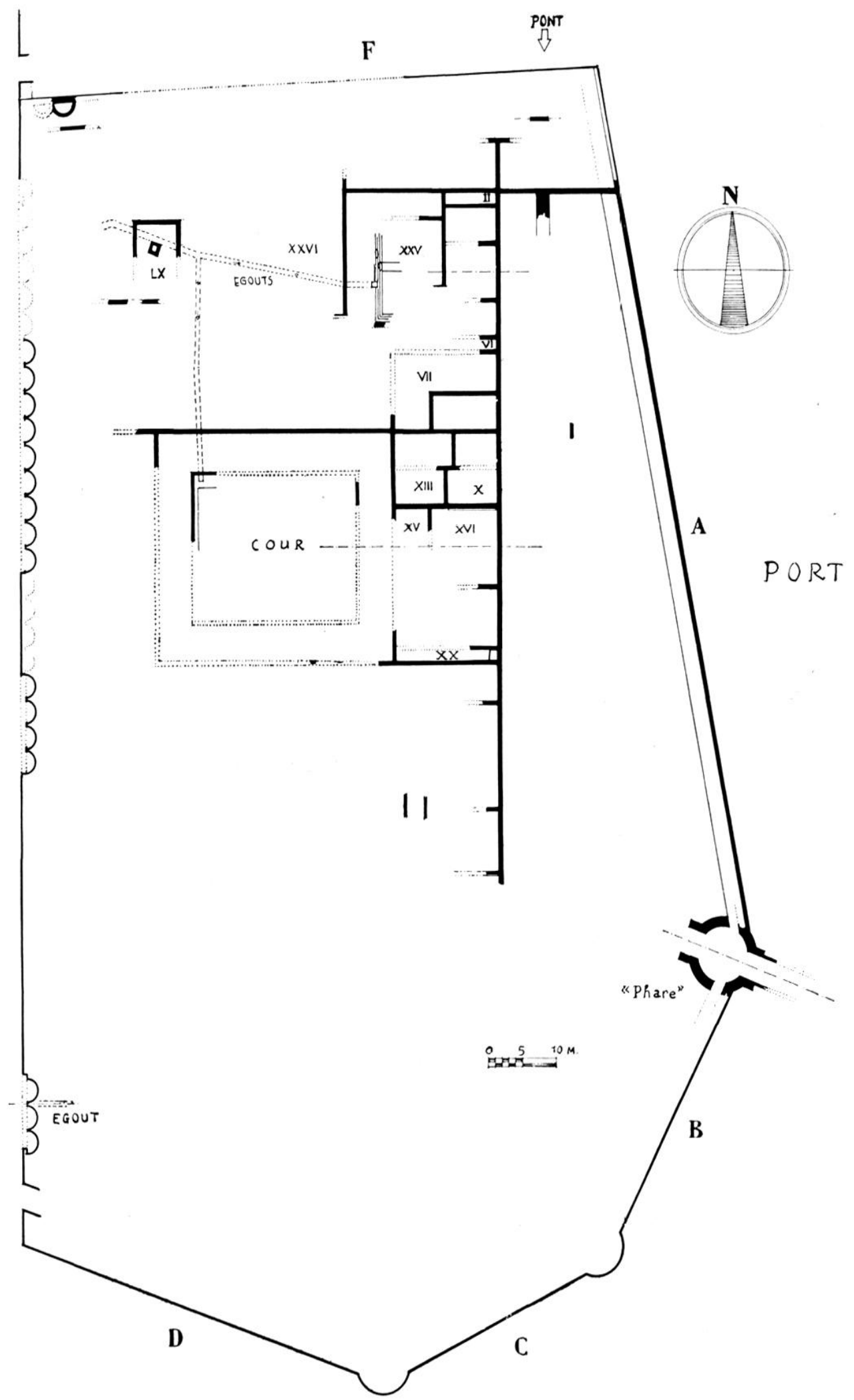

Fig. 3. - Plan de la citadelle de la Butte Saint-Antoine après les fouilles de 195̃). Le tracé des murs B, C, D et $\mathrm{E}$ a été sommairement indiqué, pour donner l'aspect général de la citadelle. (Plan dressé et dessiné par G. Giger, arch. D. P. L. G.). 
relalivement bien connues. Il est formé d'une serie de niches semi-circulaires dont l'ouverture est lournée vers l'extérieur de la riladelle et d'un mur droit plare en arant de res niches loujours ver's l'exterreur. c'est-i-dire vers l'onest. Texier a indigur du cote interieur un autre mur droit silué a 6 metres en retrail. llais il nest pas reetain, a supposer qu'il coure sur loute la fare ouest, fu'il ail joue un ròle pour retenir la terre. selon le mime archéologue. l'espace entre
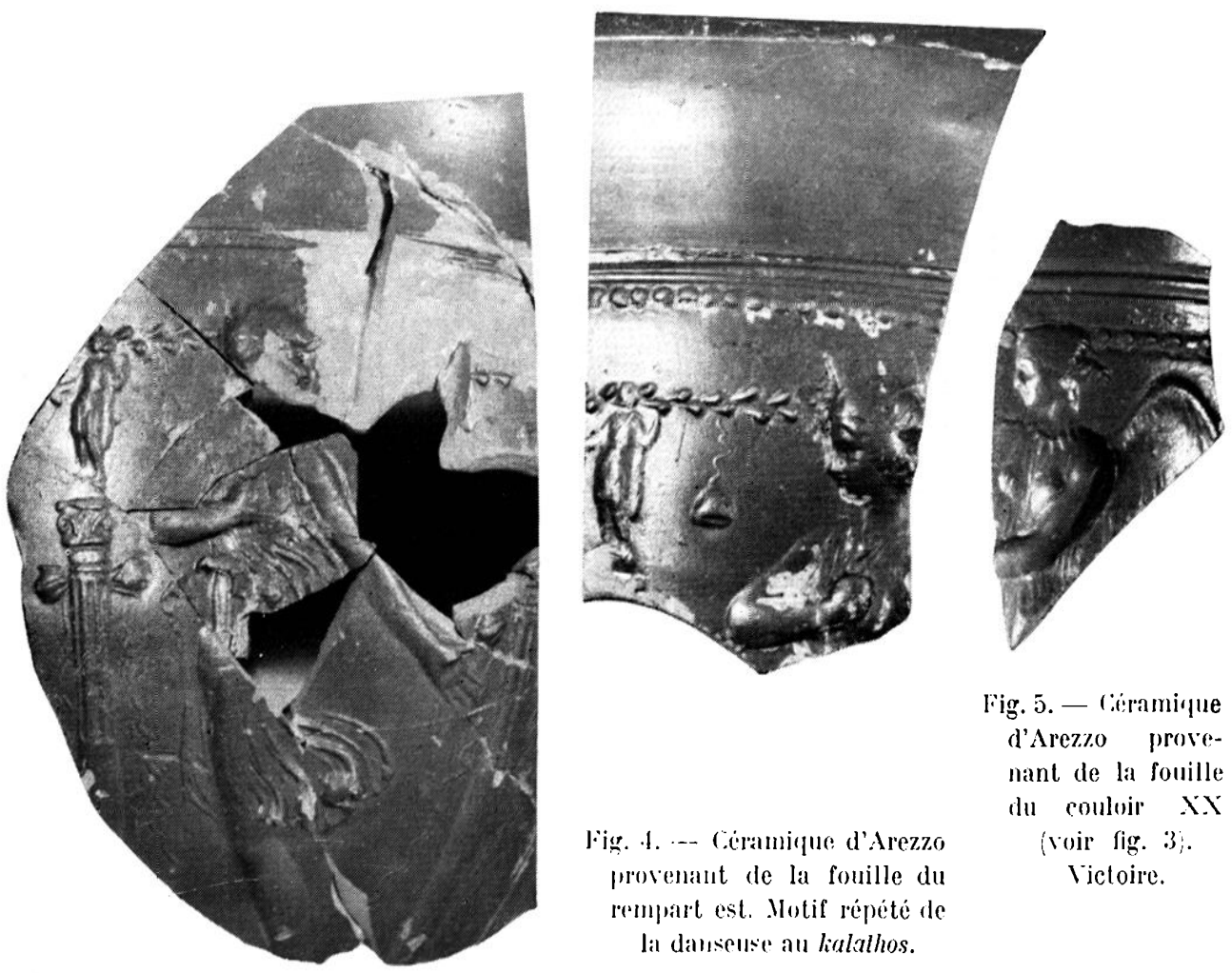

Fïg. 5. — liraminue d'Arezio provenant de la fouille du couluir $\mathrm{XX}$

Jïg. 4. -.. Ceramique d'Arezzo provenant de la fouille du rempart est. Wotif répété de froir fig. 3i. Victoire.

res murs aurail été comblé de sable marin ${ }^{8}$. Ces murs, bàtis en petit appareil régulier dont les assises ont de 9 a 10 centimètres de hauteur, reposent sur le rocher qui a été régrularisé au pic pour ne point offrir de saillies. Dans la partie méridionale est percée une porte qui paraît bien être de la mème époque; mais il faudrait pratiçuer des sondages pour ronnaitre ses dispositions intérieures confuses. Peu au nord de cette porte, débouche un égout voùté.

I a structure du mur nord (F du plan fig. 3) a pu être étudiće non loin de sa rencontre avec le mur ouest. Comme celui-ci, il est constitué d'une série de niches semi-circulaires fermées par un mur plat épais de $0 \mathrm{~m}, 80$; les niches ont 3 mètres de diamètre intérieur et leurs parois ont $0 \mathrm{~m}, 60$; les moellons sont hauts

(8) (h. Texier, art. cit., p. 187. 
de 10 centimètres environ (figr. 6). En arrière, à 3 mètres des niches, est un mur épais de $0 \mathrm{~m} .60$ ), de technique identique. La plupart des murs du terre-plein de la citadelle ont cette épaisseur. L'intervalle entre les niches et le mur de facade n'était pas comblé à l'époque romaine : à 2m.5) au dessous des dernières assises conservées a été découverte, sous une épaisse couche de gravats, de la céramique vernissée jaune ou verte de la fin du Hoyen Age ou d'une époque plus récente. Il est vraisemblable qu'elle a été déposée là soit lors du creusement du canal des Moulins, soit lors de la fortifiration de la butte, dans la seconde moilié du $\mathrm{XvI}^{\mathrm{e}}$ siècle. Il en étail sans doute de même dans les autres niches.

En revanche, l'intervalle entre les niches el le mur intérieur élait comblé à l'éporque romaine : la tranchée est tombée sur des terres non remaniées qui ont servi à niveler la ciladelle. Deux couches de déblais d'environ 1 mètre d'épaisseur chacune, séparées par une couche sterile de $0^{\mathrm{m}}, 90$, ont été fouillées. La première de ces couches élait recouverte d'une bande de sable qui s'appuyait contre la paroi exlérieure de la niche. Il n'apparaill pas de différence dans la céramique des deux couches. Il en provient, outre des enduits peints, de la céramique campanienne $B$ et C et tardive, dont quelques fragments des formes $1,2,3$ el $31^{9}$. Les rebords bien caraclérisliques de la céramique aréline appartiennent au service I de Haltern, différencié par la lèvre pendante ${ }^{10}$. Deux fonds mal conservés portent les marques $\frac{\text { Vil }}{\text { SCR }} 11$ et ROIL(?). La couche supérieure rontenait de la vaisselle à parois fines : un fragment est décoré de pommes de pin ; un autre a une frise de feuilles de lierre et porte en relief les lethres IAN ${ }^{12}$. Il s'y ajoute un fragment de lampe delphinoïde et un bec en forme d'enclume.

Le mur nord est en partie détruit ; il n'en reste que les extrémités orientale et occidentale. Celte dernière s'est conservée, car à basse époque deux salles voûtées ont été accollées contre elle. A l'est, la présence d'un pont faisant communiquer la citadelle et la ville a protégé un autre pan. L'existence de ce pont s'explique par celle d'un fossé isolant la citardelle de la cité ${ }^{13}$.

(9) X. Lavibogla, Par una classificazione preliminare della ceramica campana, dans Alli del $1^{\circ}$ congr. di sludi lig., Bordighera, 1952, p. 139-206.

(10) S. LoeschCk1, Keramische Funde in IIallern, dans Mill. der Alleriumskiomm. für I1'est/alen,

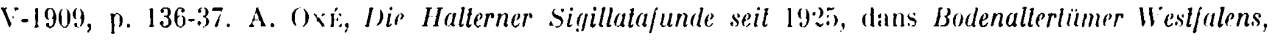
VI.1913, pl. I.

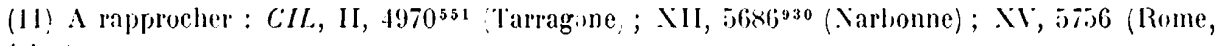
Farnésine).

(12) J'ai du mal a restituer Buccio Norbatius connu en Italie, Autriche et Viugoslavie, selon II. Ohlenroth. Voir J. DÉcmeterte, Les vatses ceramiques ornes de lit (inule rom., I-1901, p. 10, fig.

(13) Sur la question de savoir s'il y avait un canal a l'éporque romaine. p,ur anener l'eau dans le port, une discussion a eu lieu entre MI. Donnadieu et Formige. Il ne sera possible d'apporter de nouveaux arguments qu'après des fouilles. Notons qu'il y avait aussi un fossé il la Plate-lorme vers le nord, comme l'ont montré de récents travaux 


\section{II. - Les constrections de L'NTtérielr}

Sur le terre-plein ainsi délimité, des murs placés avec régularité avaient èté signalés : les fouilles ont confirmé leur existence. Cependant, si le plan donné par Texier est exact, il faudrait penser qu'il y avait des irrégularités dans la partie méridionale. D'autre part, un espace peu ou point construit se remarque le long du mur oriental.

Les murs mis au jour suivent l'orientation nord-sud de la muraille occidentale de la citadelle et les constructions paraissent se grouper autour d'une cour centrale à promenoir ou à portique (fig. 3). Le mur nord du promenoir de cette cour a été suivi sur toute sa longueur : il mesure $34 \mathrm{~m}, 10$ de long, le sol est constitué d'une mince couche de ciment posée sur la terre. Le côté oriental devait avoir $: 99 \mathrm{~m}, 50$, s'il est bien vrai que son angle sud-est a été retrouvé à l'extrémité du couloir XX. D'autre part, une partie du mur oriental qui sépare le promenoir de la cour a été retrouvée, ainsi que l'angle nord-ouest de ce mème mur. A ce point, de la cour part un égout orienté sud-nord, qui va se jeter dans un autre égout orienté est-ouest. Le sol de la cour, près de cet angle, est identique à celui du promenoir.

A l'est de cette cour et au nord, sont venus au jour plusieurs salles rectangulaires et des couloirs, qui ont parfois conservé leur sol. Il est impossible de reconnaitre la destination de chacune de ces pièces; seuls les couloirs se distinguent par leur étroitesse.

lin sondage a élé fait, à l'est, dans le couloir $X X$ qui unissait la zone orientale non bâtie à la cour centrale. Sous la couche de terre grise de surface, s'étendait une couche argileuse jaunâtre, non remaniée : une terre idenlique a été retrouvée sous le sol de la salle XV. A la base de cetle couche, sur le rocher, reposaient des fragments de poterie arétine el campanienne el surtout des morceaux de vases à parois fines avec des décors soil d'incisions verticales ou horizontales, soit de pommes de pin. Il en vient aussi une olla en terre noircie et deux petits bronzes d'Intibes, datés de 44-42 avant notre ire : ils portent une tête de femme tournée à droite avec légende $\triangle H M$; au revers, une victoire couronnant un trophée, avec légende $\Lambda E \Pi^{14}$. Une monnaie de ce lype a déja été découverle à Fréjus, lors des fouilles du quartier du marché par le I)r Donnadieu ${ }^{15}$, ce qui tend a montrer que ce type de monnaie avait cours hors d'Anlibes. Dans ce couloir, à son extrémité orientale, a été creusée une fosse qui fut ensuite remplie de c'endre. Outre de nombreux coquillages, on y a trouvé un fragment de vase d'Arezzo,

(14) A. Blaxchet, Trailé des monnaies gauloises, p. 442 ; F.-R. Varaldi, A propos d'Antibes et de ses monnaies, dans Ann. de la soc. scient. et litt. de Cannes, IX-1937/38, p. 139-148; M. Graxt, From Imperium to Auclorilas, Cambridge, 1946, p. 390.91.

(15) Paris, Bibl. doc Mon. hist., ms. Donnadieu, 2, rapport de 1930. 
décoré d'une Victoire lournée à gauche de l'atelier de Perennius ${ }^{16}$ (fig. 5). La fosse est sans doute postérieure à la construclion des murs ; quant à la couche de terre jaunâtre, je ne suis pas certain qu'elle soit anlérieure aux murs du couloir el il me parait plus vraisemblable de supposer qu'elle a été placée immédiatement après leur construction. Ilais pour confirmer cette hypothèse, il faudrait des sondages de l'autre còté des mur:s.

Plus importante pour la datation a été la fouille effectuée, toujours à l'est de la cour, entre les salles X-XIII et XV-XVI. Dans la salle $X V$, le sol de béton

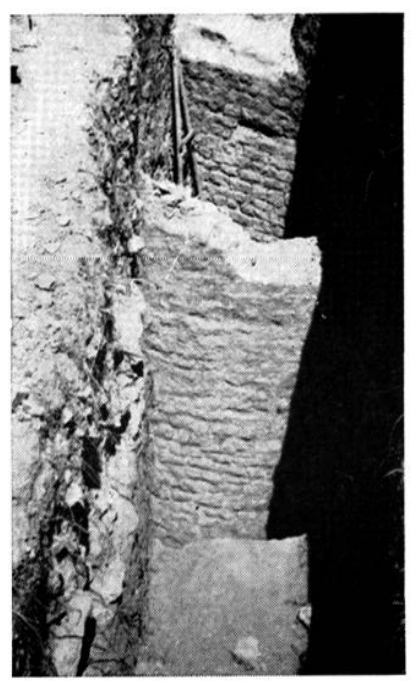

liig. 6. - Sondage au revers du mur Nord, vu du Sud. Au fond, le mur de façade; en avant, le mur du contrefort semi-circulaire, vu du côté convexe.

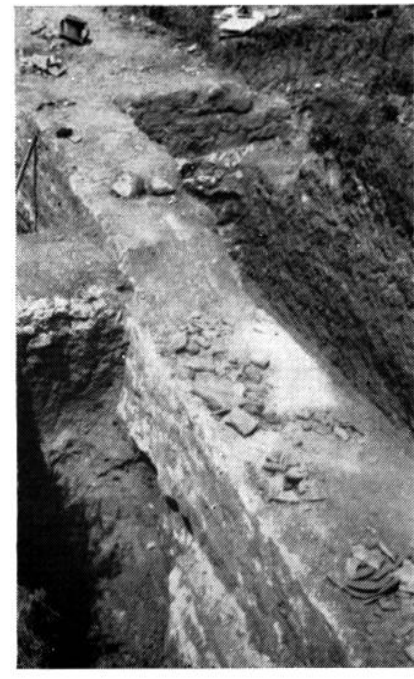

Fig. 7. - A gauche, la salle XVI, vue du Sud-Est; à droite, la salle $\mathrm{X}$.

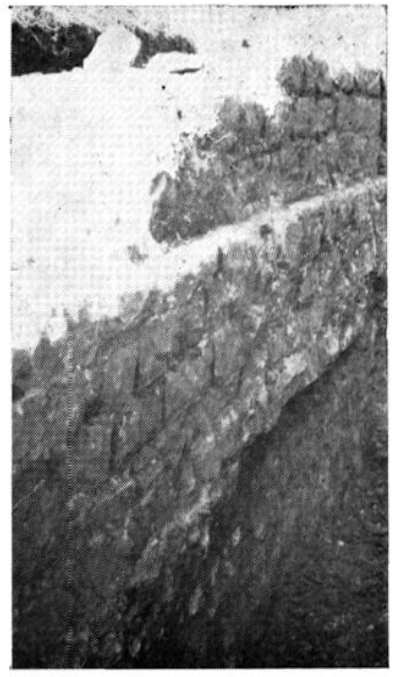

Fig. 8. - Mur séparant les salles $\mathrm{X}$ et XVI, vu du Nord-Est. De bas en haut: béton de fondation, mur en appareil assez irrégulier caché jadis sous le sol, décrochement au niveau du sol, mur au-dessus du sol.

s'est conservé ; au dessous, une couche d'argile jaunâtre d'environ 20 centimètres, mêlée de chaux; plus bas, au-dessus du rocher, une terre argileuse rougeâtre, contenant de la poterie du type d'Aco, à grandes pointes effilées, un rebord de vase d'Arezzo (Dragendorff 7 ?) du service I et un fragment de campanienne (C. Par comparaison avec ce qui se passe dans les salles X et XVI, il semble que l'argile rougeâtre soit antérieure aux murs. En effet, entre ces deux salles, comme il ressort de l'étude du mur et des couches, la tranchée de fondation a été creusée jusqu'au roc, sur une profondeur de $0^{\mathrm{m}}, \overline{5} 0$ dans une terre argileuse assez compacte ; les fondations ont été bâties en blocage. Au-dessus, le mur en appareil irrégulier à joints très débordants, qui fait saillie au-dessus des fondations, a été

(16) II. Dragiendonff, op. cit., p. 64.65 et pl. I, notamment nos 7, 8 et 13. 
ronstruil à l'air libre et remblacé immédiatement après la construction par une argile jaunatre compacte semblable à celle du couloir $\mathrm{XX}$, dans la salle $\mathrm{X}$, et par une terre plus sableusc, dans la salle XVI. Dans cette dernière, sur cette couche a été posé le sol de béton. I)ans la salle $X$, le sol ne s'est pas conservé, mais il devait reposer sur l'argile jaunâtre (fig. 7 à 9). C'est de la couche antérieure à la construction du mur que viennent les poteries. Il y a de la campanienne $\mathrm{B}$ et $\mathrm{C}$ et de la campanienne tardive dérivée de $\mathrm{A}$, de couleur brune et décorée de cercles concentriques blancs (forme 31). La poterie arétine est représentée par un fragment de plat à rebord uni, des rebords de ce type el

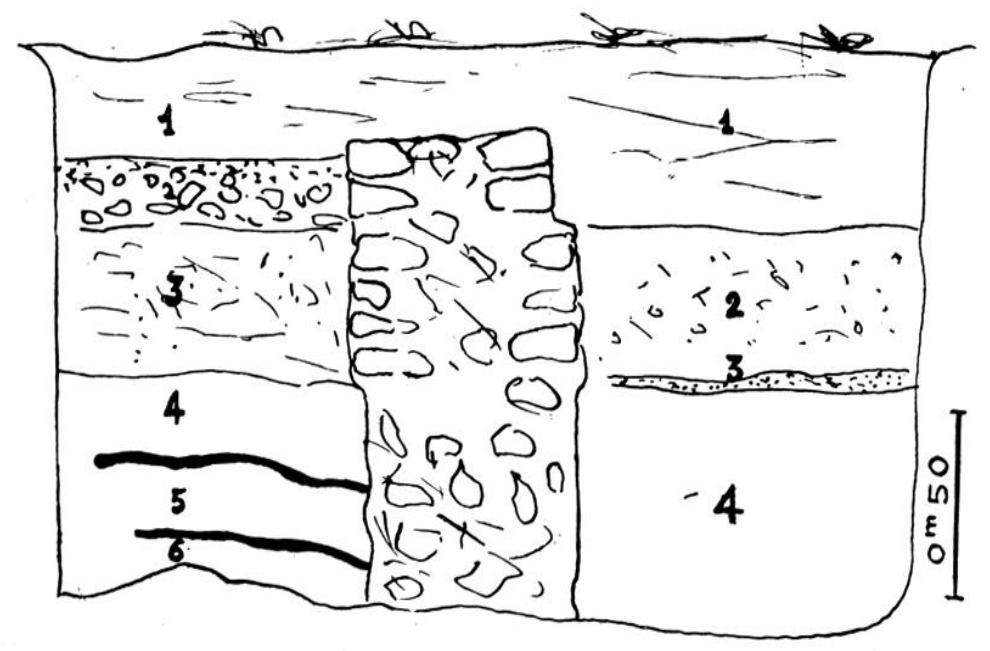

Fig. 9. . . ganche, salle XVI (1 terre remaniéc, 2 sol, 3 argile ct sable, 1 argile brune, 5 foyers, 6 argile blanche; $a$ droile, salle $X$ ( 2 argile jaunitre, 3 sable, 4 argile lirune:

d'autres à lèvre pendante. En outre, des fragments de poterie fine du type d'Aco, des becs de lampe à tête d'oiseau et un col d'amphore avec: une inscription en rouge tracée au pinceau... VS/RO (fig. 16, 18). Au sud du mur, dans la salle XVI, a jo mètres de l'angle nord-est ont été localisés deux petits foyers superposés; le plus ancien reposait sur une mince couche d'argile blanche. Entre les deux. quelques tessons de campanienne tardive el un plat à engobe rouge intérieur. Ces deux foyers représentent la trace d'un habital antérieur à la construction des batimenls intérieurs de la ciladelle.

Au nord-est de la cour centrale, la salle VII avail conservé une partie de son sol en béton posé sur un radier de pierres irrégulières.

La couche la plus basse a été très pauvre en céramique; au-dessus, une couche de sable fin avec de la céramique identique à celle de la couche supérieure. Celle-ci, formée de sable et d'argile, contient de la poterie d'Arezzo dont un fond avec la marque PIIER'T' 


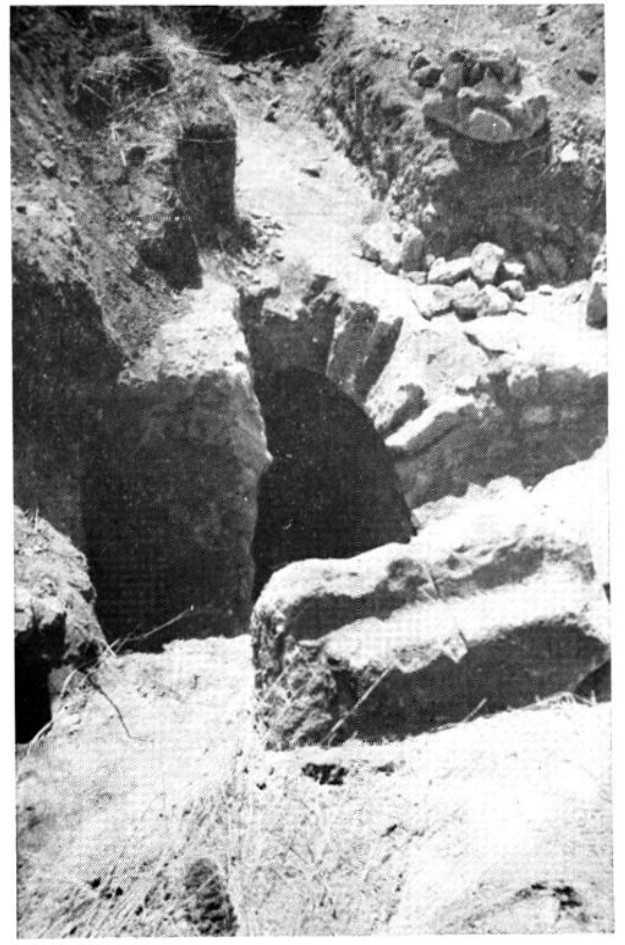

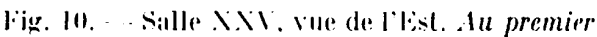
plan, canisianu: an secend plan, depart de ligout orienti est-ouest.

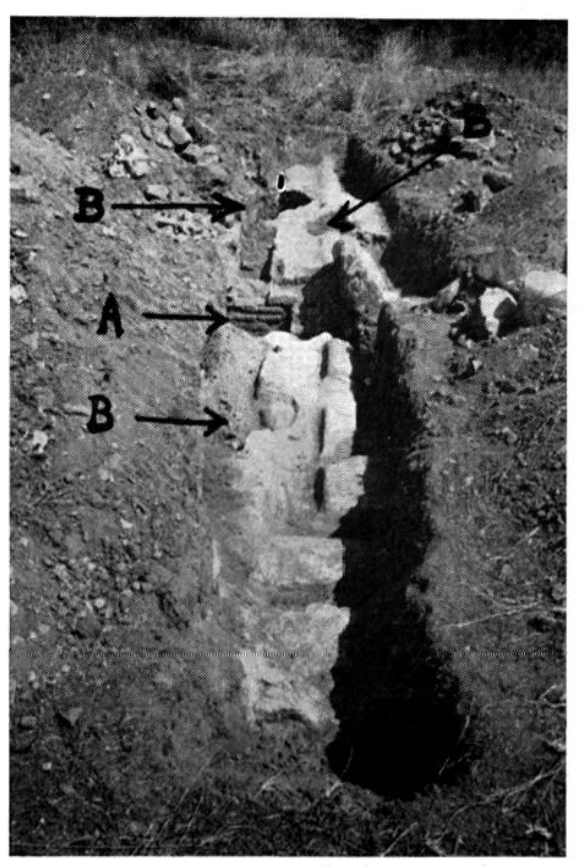

Fig. 11. - Caniveau de la salle XXl. En $A$, cgout ; en $B$, silos (?).

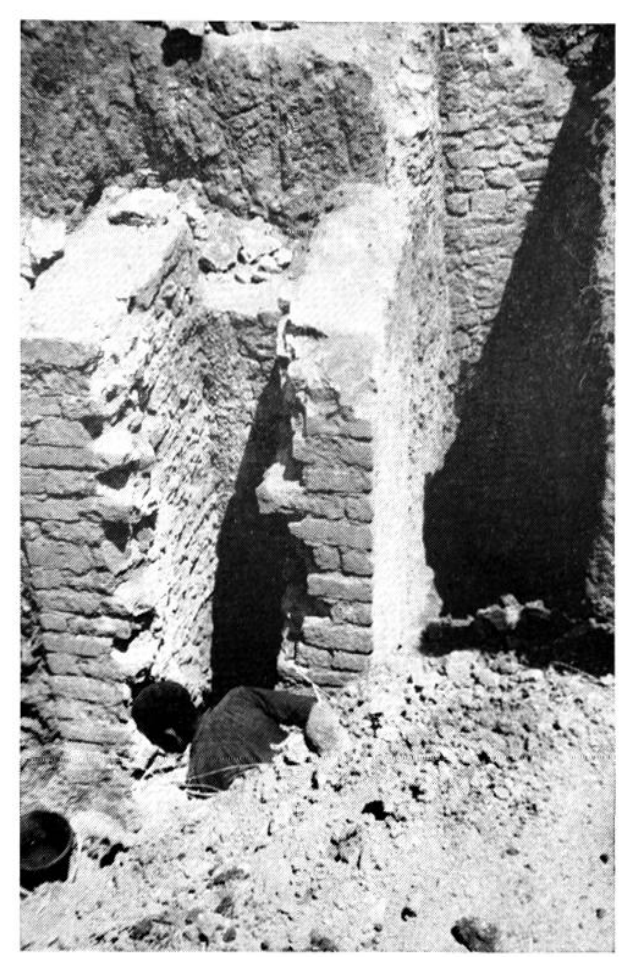

Fïg. 12. - P'uisard de la salle I.X, vu dul sud. llu fond, te mur nord de la sille.

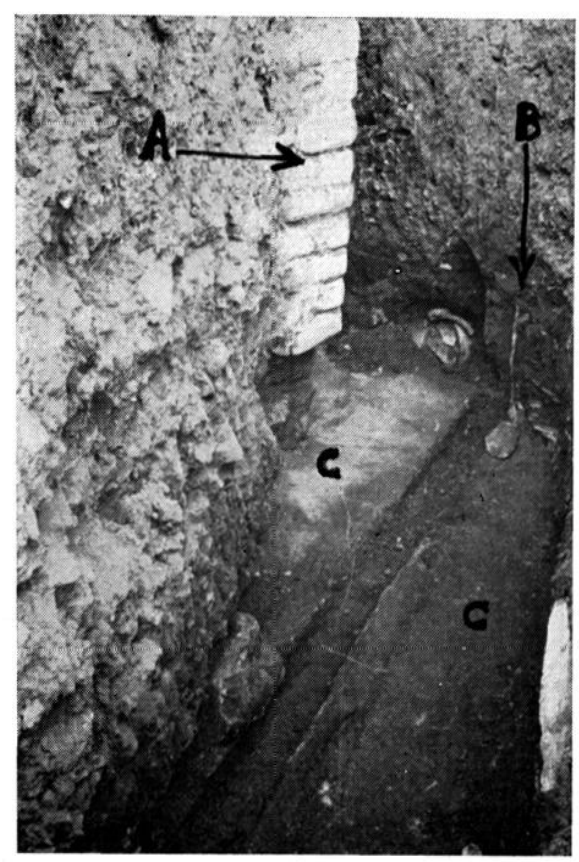

Fig. 13. - Salle IX. En A, angle Sud-list du puisard; en $B$, enduit peint en place; en C., sols, siparés par la trace de l'ancienne cloison. 
avec ligalure PHE ${ }^{17}$, de la campanienne tardive el de nombreux fragments de poterie fine. Ces derniers offrent soit des incisions paralleles, soit de petites pastilles rectangulaires disposées en filets ${ }^{18}$, soit un décor de pommes de pin, soit des pointes du type d'Aco. Ajoutons des becs de lampe à tête d'oiseau. Je ne peux alfirmer que cetle dernière couche, sur laquelle repose le sol, est antérieure au mur de la salle; la présence d'une zone charbonneuse qui pourrail être un ancien foyer le ferait croire. L'étude de cette salte a été compliquée du fait que le sol a été crevé à une date lardive, qu'une partie de la terre argileuse a été extraile et que, par la suite, le trou a été comblé avec une terre grise conlenant de la poterie grise estampée à décor de palmelles, des vases en terre grise rugueuse el une boucle de ceinturon en fer, de forme ronde.

Au nord de la salle VII, les tranchées ont mis au jour d'autres salles et couloirs, parmi lesquels la salle $X X V$, dont la partie occidentale et le mur oriental ont été dégagés. Ce doit être une petite cour rectangulaire à laquelle on accédait par les couloirs II et VI. Le mur ouest et une partic au moins du mur sud sont longés par un caniveau creusé dans des dalles de grès tendre; contre le mur est, une rangée de dalles de grès. Le caniveau déversait ses eaux dans un égout orienté est-ouest (fig. 10). A une époque inconnue, entre le $\mathrm{I}^{\mathrm{er}}$ et le $\mathrm{IV}^{\mathrm{e}}$ siècle, ('e caniveau a été coupé par des fosses d'ouverture arrondie; deux ont été creusées et une seulement ébauchée. Peut-être s'agit-il de silos. A une époque plus récente (fin du $\mathrm{IV}^{\mathrm{e}}$ ou $\mathrm{v}^{\mathrm{e}}$ siècle), partie du caniveau et un des silos ont été recouverts d'un mur : entre ce mur et le caniveau, on a trouvé un rebord de terre sigillée claire et un morceau de panse de vase en terre grise estampée à décor de palmettes (fig. 10 et 11 ).

A l'époque de la construction de ce mur, l'égoul a été comblé. Large de $0^{\mathrm{m}}, 60 \mathrm{et}$ haut, à son départ, de 1 mètre, il est construil en petit appareil régulier à joints débordants et aplatis. Les moellons sont hauls de 9 à 10 centimètres et longs de 10 à 25. Le fond est cimenté et présente une petite rigole médiane. La pente de cet égout n'est pas uniforme, et il en est de même pour l'égoul sud-nord qui le rejoint. $\Lambda 23$ mètres de la cour XXV, après n'avoir eu qu'une faible pente, il descend brusquement sur 1 mètre; puis sa pente diminue et, a l'ouest du puisard. s'accroìt de nouveau. Cette irrégularité est due à la forme des bancs de grès. Pour la visite de l'égout, un puisard avait été aménagé à 29 mètres du départ. Sa section intéricure est un carré de $0^{\mathrm{m}}, 60$ de còté. Dans ses murs ont été ménagés des trous facilitant le passage (fig. 12). La fouille de cel égoul, inachevée, a donné en plus d'un fond d'Arezzo avec la marque $\frac{\text { BVGCIO }^{19}}{A N G H}$

(17) A développer sans doute en P. Hertorius: CIL, II, 4970225 (Tarragone); V, 54 (Gênes); $\mathrm{XI}, 6700^{319}$; XII, $5686^{390}$ (Genève) ; XV, $5256 \mathrm{k}$ (Rome). Voir S. Vevtura Solsona, Las marcas aljareras de la "terra sig." halladi en Tarragona, dans Mem. de los mus. arq. prov., 1948-1949, p. 158 et fig. 58 . n. 2126 .

(18) $\Lambda$ rapprocher : M. Labrocsse, Les fouilles de Gergovie, dans Gallia, VI-1948, p. 84, fig. 34,

(19) CIL, II, $4970^{91}$ (Tarragone) ; XV, 4958 (Rome, Farnésine). 
une très grande quantilé de vases en terre sigillée claire des II $^{\mathrm{e}}$ el III $^{\mathrm{e}}$ siècles, de la poterie estampée chrétienne, dont un fragment de lampe, rouge et grise, ornée de grilles, de palmettes stylisées et de rouelles. Il faut ajouter des cubes de mosaïque, des verres et une fibule en fer. Sur le fond, a été relevée une monnaie de Valentinien I, du type de la victoire ailée avec la légende Securilas rei publicae ${ }^{20}$.

$A$ l'ouest de la cour XXV, à l'angle sud-est de la salle $X X V I$, un sondage a donné, au-dessous de la couche de surface, une terre blanchàtre mèlée de cendre, contenant un fragment de vase de la Graufesenque à décor d'oves, de la sigillée claire et une luile avec marque L. HEREN.N. avec les ligatures HE et $N \mathrm{~N}^{21}$. Au-dessous, une lerre argileuse brunâtre avec de la poterie d'Arezzo, de la campanienne $A$ tardive, B et C. La poterie fine est de types variés: incisions, décors de marguerites ou de cercles en relief, pointes effilées. Il faut ajouter une vaisselle ocre ornée de rinceaux peints en rouge. Cette couche qui repose sur le sable est peut-être postérieure à la construction des murs.

Plus à l'ouest, a été fait un sondage important pour l'histoire des débuts de l'occupation de la butte. La salle rectangulaire $L X$ et le puisard signalé plus haut ont commencé d'être dégagés. Or à ce point le rocher est très bas et les constructeurs, pour établir le terre-plein de la citadelle, ont dû effectuer un important remblaiement. Le rocher est à 4 mètres du sol actuel et à $2 \mathrm{~m}, 30$ du sol de la citadelle. Le sondage a montré que le mur ouest de la salle LX et le puisard avaient coupé une construction antérieure : à 2 mètres sous le niveau gallo-romain de la salle $\mathrm{L} X$, ont été trouvés trois sols en béton de tuileau, juxtaposés, appartenant à une maison (fig. 13). Malheureusement lors de la construction de la citadelle, les pierres des murs extérieurs ont été arrachées et réemployées et il n'est resté que les sols. Ces sols devaient être séparés par des cloisons de bois : aucune trace de mur, en effet, n'a été retrouvée, même en fondation; d'autre part, à l'angle d'un de ces sols, s'était conservé en place un enduit peint rouge, qui, au revers, portait la trace des lattes de bois contre lesquelles il s'appuyait. Ces lattes étaient montées en épi (fig. 14). Sur ces sols ont été jetés, lors de la destruction, de nombreux morceaux d'enduits peints et des poteries provenant sans doute de la maison. En voici un inventaire sommaire :

bol de campanienne tardive (forme Lamboglia 31),

bol d'Arezzo (Dragendorff 7, type 7 de Haltern), avec marque $\frac{A \text { VIBI }}{\text { DIOMED }}$ el graffite

(20) II. CoHEx, Descr. hist. des monnaies..., VIJI, p. 92, nº 37.

(21) La fabrication de ce tuilier ou de sa firme parait essentiellement réduite i la côte provencale et ligure et aux abords : CJL, V, $8110^{445}$; XII, 567944. Carle arch. de la Gaule, II (Var), 16 D, 28, 40, 174; V (Bouches-du-Rhône), 75, p. 34. X. Lasi Bofilit, Gli scavi di Albintimilium..., Bordighera, 1950, p. 158, 178,195 . Fréjus est particulièrement riche : il faut ajouter une marque sur antéfixe découverte dans une citerne avec de la sigillée claire et une tuile plate du mème atelier. Il convient de se demander si Fréjus, qui possċie de riches gisements d'argile utilisés au Moyen Age, n'ítait pas un centre de la production de tuiles romaines aux $\mathrm{II}^{\mathrm{e}}$ et $\mathrm{II \textrm {I } ^ { \mathrm { e } }}$ siècles. 
au fond exlerieur PIII, appartenant au service I de Hallern, comme les aulres fragment. ${ }^{22}$,

fond de coupelle avec marque. $\mathrm{NC}^{23}$.

grand plat (Dragendorfl 1, type 1 de liallern) avec marcue LSF,

fragment de grand plat avec marque sur les bords du fond $\frac{\text { IIL.ARVS }}{\text { L. LE(GIDI }}$ (fig. 15)),

fragment de gobelet en argile ocre, avec en relief sur la panse HILAliVs ACO; il est orné de petites poinles effilées ${ }^{25}$.

nombreux cols d'amphores, dont un avee marque sur la livere B..... ; plusieurs ont une pâte trìs micacée ${ }^{26}$,

deux bouchons d'amphore en argile, dont un avec deux tridents (fig. 16).

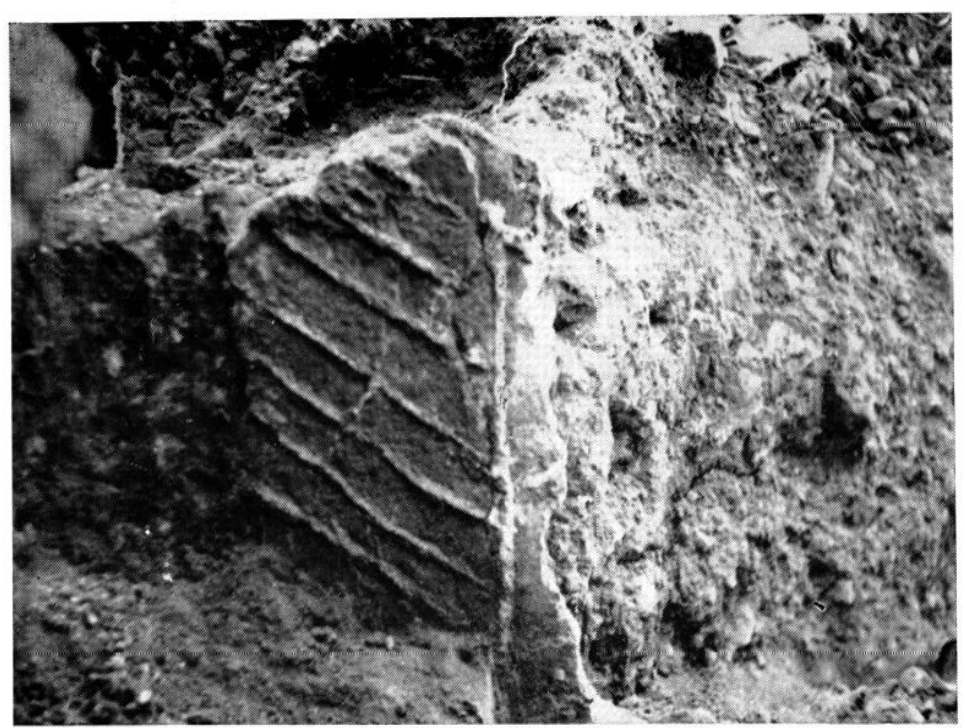

Fig. 14. - Salle I.X. Enduit peint. vu du revers, avec la trace des lattes.

Ce dépôt de poterie est sans doule postérieur à la construction des murs de la salle LX el du puisard : ces murs sont tries bien appareillés et, lors de la fouille, il a élé

(22) CIl, XI, 6700, 7690; XII, 5686692.9 (dont une ì Fréjus): XIII, 10009, 300 (Neuss). Chr. Arbrecits, Das Römerlayer in Oberiaten, $\mathrm{II}^{1}$, Dortmund, 1938, p. 56, no 42 ; $\mathrm{II}^{2}, 1942, \mathrm{p} .16$.

(23) CII., XI, 670)1019 (Arezzo); XV, 4956 (Rome).

(24) Autres marques de l'oflicine de L. Legidius: CIL, XI, 6700, 324-26 (Arezzo); XIII, 10009 143-144 (Ncuss); XV, 5270-5272 (Rome).

(25) Sur la production d'Aco, M. OhJenroth prepare une étude. Voir J. J)Fculistre, op. cil., I, p. 31-41. Sur Irilarus en particulier : CIL, XII, $5686^{6}$ (Orange). Chr. A t.3nicirs, op. cit., $\mathrm{II}^{1}$, p. 46-47; II ${ }^{2}$, p. 46-17 et pl. 4. R. EgGi:R, Die Alusgrabungen auf dem .Magdalensberg, 1951, Klagenfurt, 1954, p. 40 et fig. 20, extr. de C'trinthin, I-143. Sur l'origine et la biblingraphie, Storin di Milann, I, Le origine e l'elit romiana, 1953, p. 1\%x, n. 4 et p. 185.

(26) Chr. Albrecits, op. cit., II ${ }^{2}$, p. 82-87 et 95-97. Comparir avec les reburds d'amphores de

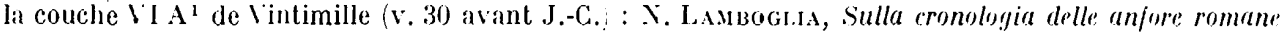
di elà reppublicana, dinns Rir. di Studi liguri, XXI, 1955, fig. 16, p. 260. 
visible que les fragments d'enduits peints sont venus se briser contre le mur du puisard. Mais comme les poteries viennent de la maison détruile (elles sont mêlées aux enduits peints qui en viennent cerlainement), elles permetlent de dater et la derniere occupation de la maison et les murs de la salle LX.

Il reste à signaler deux pelits sondages qui n'ont pu ètre achevés. Près du pont nord, la présence d'un chemin a empêché de retrouver le plan de la porte. Il a seulement été visible qu'un mur des constructions intérieures de la citadelle vient s'appuyer sur le chemin de ronde qu'il coupe. Mais l'etat des recherches ne permet pas de dire si re mur est de la même époque que le reste des murs

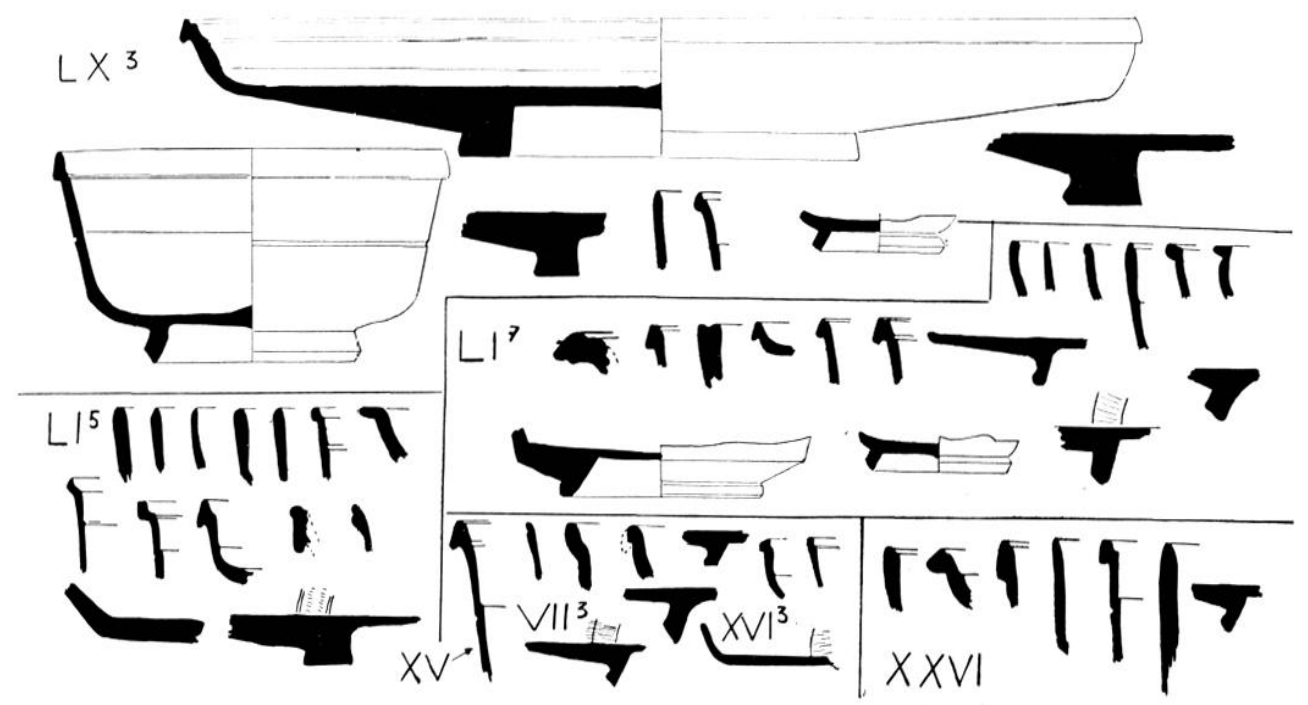

ligg. 15. -.- Cimamique d'Arezo. Échelle $1: 3$.

du terre-plein. ou si un rhangement de parti est intervenu au cours de la construction. -- Inans la parlie sud, une tranchée a fait découvrir deux murs de basse époque bâtis avec des matériaux provenant d'un édifice très soigné, en grand appareil. Ce sont des fragments d'architrave.

$$
* *
$$

De cette campagne de fouilles, il est permis de tirer un certain nombre de conclusions ; malgré leur fragilité (car des fouilles ultérieures en modifieront (certaines) elles sont nécessaires, ne serait-ce que pour diriger de futures recherches.

Tout d'abord, apparait la nette ressemblance entre le plan de la citadelle de la Plate-Forme et celui de la Butte Saint-Antoine (voir fig. 1). Les murs extérieurs épousent la forme d'un parallélogramme qui suit les irrégularités du terrain ; à l'intérieur, au contraire, les murs s'ordonnent avec régularité autour 
d'une cour centrale. Pour le moment, je ne sais si l'entrée monumentale de la cour doit être recherchée sur sa face nord ou à l'est : la première hypothèse rendrait encore plus nette la ressemblance entre les deux constructions.

Il est impossible de préciser le rôle de la butte Saint-Antoine. Le Dr Donnadieu a vu dans la Plate-Forme le prétoire, comprenant la maison du commandant en chef et des annexes, et il supposait des casernements sur la butte : cette dernière hypothèse ne semble pas confirmée par les fouilles et il est préférable de réserver toute opinion.

Vraisemblablement, les murs d'enceinte ont été bâtis les premiers, pour servir de cadre aux constructions du terre-plein. Cela est peut-être confirmé par la présence du mur qui coupe le chemin de ronde près du pont. D'autre part, la différence de technique entre les différents murs d'enceinte montre que des équipes diflérentes ont travaillé sur la hutte. Plutôt que de supposer qu'elles ont travaillé en même temps, je crois préférable d'imaginer qu'elles se sont succédé, pendant une période qui peut être assez courte. Ians ce cas les travaux auraient commencé par la construction du grand mur plein de l'est, se seraient poursuivis par les faces sud, où la construction devient moins massive, enfin se seraient achevés par les faces est et nord, caractérisées par les contreforts intérieurs ${ }^{27}$. La technique de ces derniers murs est presque identique à celle de l'égout et des murs encore conservés du terre-plein.

Forum Julii, dont la première mention est faite dans la correspondance de Plancus et de Cicéron en 43 avant J.-(i., n'a pris son importance qu'après Actium (31 av. J.-C.). Même si l'on admet, avec M. Donnadieu, que l'établissement a été un chantier naval pendant la guerre maritime contre Sextus Pompée ${ }^{28}$, on est obligé d'admettre que son développement date de l'arrivée de la flotte d'Actium, ou d'une partie de celle-ci, et de l'installation d'une colonie de vétérans de la VIIIe légion ${ }^{29}$. Mais s'il était normal que la colonie fût entourée d'une muraille, il n'était pas indispensable de la flanquer de deux citadelles, si ce n'est pendant une période de troubles. Or, jusqu'en 14 avant J.-C., date de la campagne alpestre d'Auguste, selon Dion Cassius, les populations des Alpes-Maritimes restèrent insoumises ${ }^{30}$. Parmi celles qu'énumère le Trophée des Alpes (la Turbie),

(27) A Beaucaire : Carte archéol. de la Gaule, VIII (Gard), 3, p. 3. A Saint-Jsertrand-de-Comminges: R. Lizop, Les Convenae el les Consorani, Toulouse-Paris, 1931, p. 78-79. Sur le mausolèe d'Autun : P.-M. Duval, Les portes et les mausolées d'Augustodunum, dans Bull. de la soc. nal. des Antiquaires de France: 1950-51, p. 81-87 et pl. II.

(28) La Pompéi de la Provence, Fréjus, Paris, 1927, p. 27.

(29) Rittekl.rig, s. v. Legio, dans Puuly-Wissowa, 24-1925, col. 1643. lir. Vitrixghof, Römische Kolonisation und Bürgerrechtspolitik unter Caesar und Augustus, p. 1283 et 1316, Ak. der Wiss. und der Lill., Abhandl. der Geisten-und Sozialwiss. Klasse, no 14, 1951.

(30) Drox Cassics, LIV, 24, 3. 

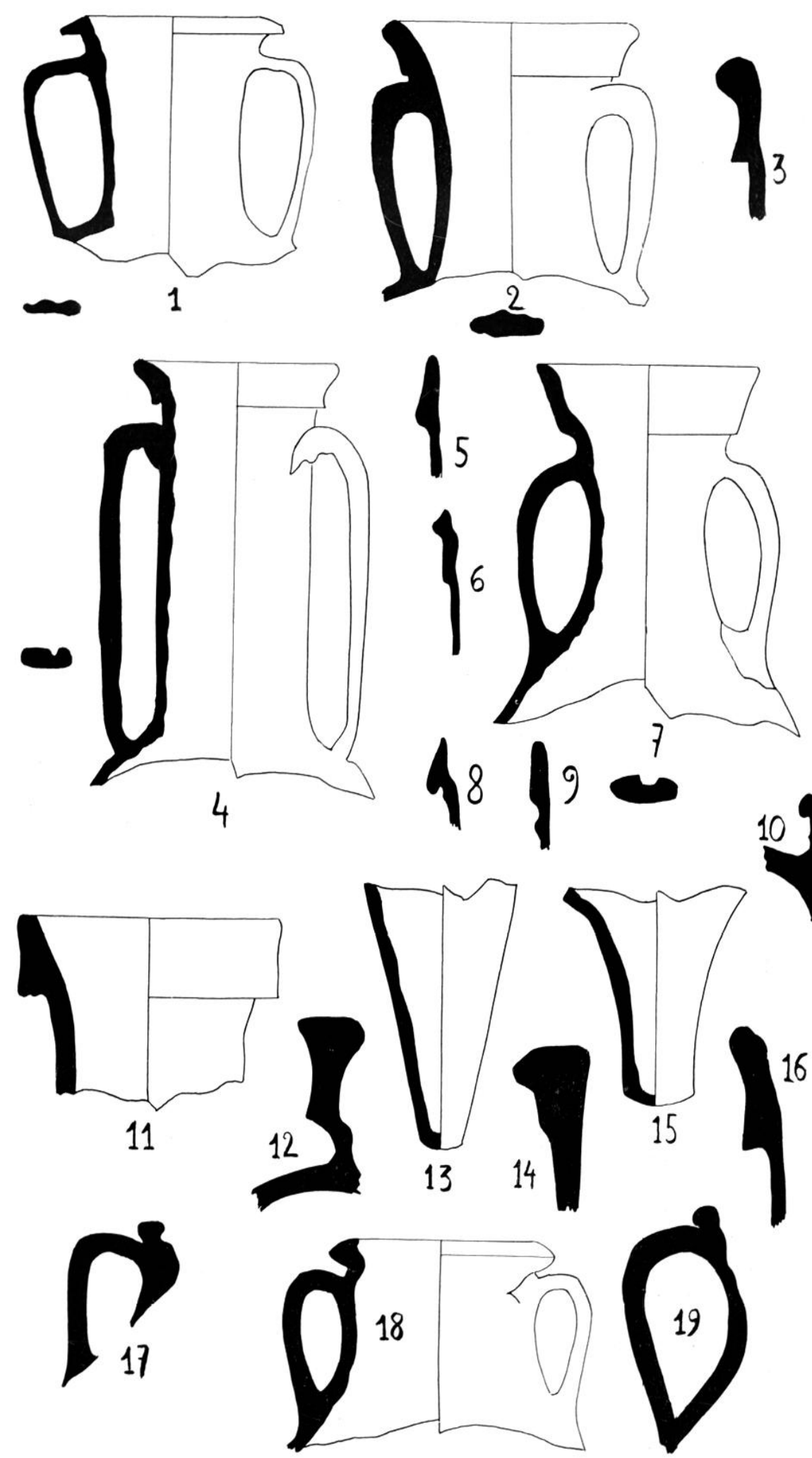

Fig. 16. -- Cols d'amphores. 1 a 4,6 et 7,10 a 17 viennent de la salle $L X, 18$ et 19 sont de la salle $X V I$ et 5 du couloir $X X .8$ et 9 ont été trouvés au revers du contrefort du mur nord. Échelle $1: 5$. 
figurent les groupes de la région de Castellane, Vergon et I)igne ${ }^{31}$. Pour les réduire, Fréjus offrait une base excellente, comme Cemenelum (Cimiez) pour attaquer reux de la région niçoise. La preuve de la pacification complète de la région est fournie par les milliaires qui jalonnent la route d'Aix à Fréjus, de Nire en Italie, et donc sans doute entre Fréjus et Nice en 13-12 avant notre ire. Je suppose done que les citadelles ont élé bâties entre les années 31 el 14 avant notre ère.

Cette datalion n'est pas contredite par l'examen de la céramique. si l'étude de la céramique décorée d'Arezzo est bien avancée, celle de la céramique commune l'est, beaucoup moins, et pour la connailre, on est obligé de se reporter aux résultals des fouilles du limes germanique. (or pour l'élude des premières productions, vers 30-25) avant, J.-C. ${ }^{32}$, ces chantiers ne peuvent donner de renseignements. L'élude de la vaisselle de Fréjus montre une triss grande homogénéité : seul le service I de Haltern, caractírisé par les livres pendantes, a été retrouvé dans les couches antérieures ou immédialement postérieures aux construclions (fig. 15). Or ce service est à Haltern contemporain de la première occupalion du camp, done des années 10 avant J.-C. Quant au service II de Haltern, qui est postérieur a 10, il est absent de Fréjus. Ionc la construrtion de la ciladelle est anterienre it celte époque. I) fail que le service I de Haltern est daté des années 10, il ne laudrait pas en conclure que la céramique de Fréjus ne peut el re assez antérieure a celle époque; il est en elfet pemis de croire que ce n'est pas la ceramique des camps du Rhin qui permetlra de dater avec précision celle de Fréjus, mais bien que celle-ci permellra de mieux connaitre les débuts de la production arétine.

L'élude du reste de la céramique permet aussi de dater la cilardelle du dernier quart, du $\mathrm{I}^{\mathrm{er}}$ sieccle avant notre ère. selon les indications de .V. L. Ohlenroth, la céramique d'Acoserait de la fin du $1^{\text {er }}$ siecles ; mais l'observation faite plus haul sur la céramique

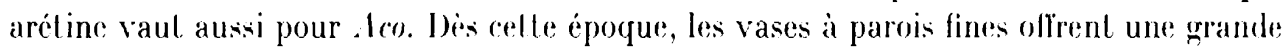
variété de motils décorés, ainsi que cela se constate à Fréjus. Quant aux lampes à lête d'oiseau, la date communément admise est l'époque d'Auguste ${ }^{33}$ el les lampes delphinoïdes peuvent ètre anlérieures ${ }^{34}$. Enfin les profils des rebords des cols d'amphore peuvent, facilement être comparés à ceux de Haltern et Oberarlen ${ }^{35}$, ainsi qu'i ceux de Vinlimille $^{36}$ (fig. 16).

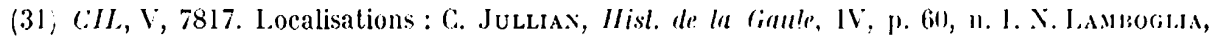
Ouestioni di top. anl. nelle Alpe mar., dans Riv. di studi lig., IX-19)13. p. 139, et carte p. 1.41.

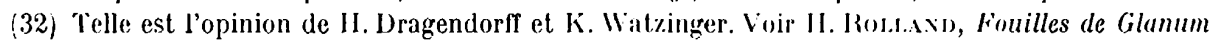

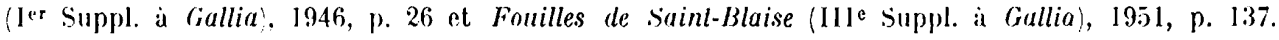

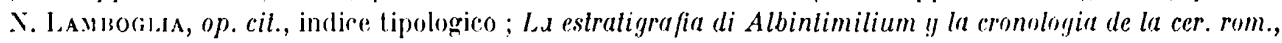
dans Ampuritas, XI-1949, p. 5.1 pet suiv.

(33) P. De l'alol Saleldas, la coleccion de lucernas rom. en el mus. arq. de Geruna, dans Mem. de los mus. arq. pror., 1948-49, p. 235-239. H. MkXzkI, Antike Lampen in römisch-yerm. Zentralmus. :u Mainz, 195.4, p. 24.

(31) 11.-B. Wat.Tens, Cal. of the Greet and Rom. lamps in the British .Mus., Londres, 1914, p.41-15.

(35) Outre les travaux citès : 1.. Cross, Gefässt!!pen der römischen Keramik im Rheinland (Beihe/t I der "Bonner Jahrbücher"), 1950, p!. 59-61.

(36) Ce sont les objets venant de la couche $V$ de Vintimille qui sont comparables a ceux de lirejus. Voir l'ouvrage rite de II. Lamboglia. Sur les fouilles récentes, il n'y a pas encore de travail d'ensemble. Lorsque les fouilles de liéjus seront plus avancées, il sera profitalite de comparer leur céramique it celle du musée de Vintimille. 
Il est surprenant de ne pas avoir trouvé, au moins en surface, un certain nombre de poteries du Sud de la Ciaule. Faudrait-il en déduire que la ciladelle a été abandonnée ¿ l'éporgue de retle production? Cela ne peut-être assuré. Celte absence est d'autant plus significative que la terre sigillée claire, dont cerlaines formes peuvent dater du ${ }_{\text {II }}{ }^{\mathrm{e}}$ siècle, ne manque pas. Les sondages montrent qu'apris celts époque, une nouvelle occupation de la butte a eu lieu, sans qu'il soil possible d'en préciser le caracire.

Au terme de la campagne de fouilles 1950 , il est done permis de proposer les hypothèses suivantes : dans la seconde moitié du $I^{\text {er }}$ siècle avant notre ère, el sans doute seulement après 31. les Romains occupèrent la Butte Saint-Antoine avec des constructions provisoires. Entre 31 et 14, ils édifièrent une citadelle protégeant le port. A l'intérieur, diverses constructions dont le ròle précis est inconnu, se groupèrent autour d'une cour centrale. Après l'époque d'Auguste, il n'y plus de trace d'occupation jusqu'au $\mathrm{II}^{\mathrm{e}}$ siècle. Ces fouilles permettent donc de montrer le ròle important joué par Auguste et peut-ître par Agrippa, dans l'histoire de Fríjus et de la Provence.

Paul-Albert Fívirir. 